\title{
What is the future of pharmacogenomics in pain management?
}

\author{
“...few knowledge resources are attempting to cross the 'omic chasm" \\ of variant interpretation and delivery of evidenced-based clinical \\ recommendations."
}

First draft submitted: 10 November 2016; Approved for publication: 10 November 2016; Published online: 19 December 2016

Keywords: chronic pain • clinical translation • guidelines $\bullet$ pharmacogenomics

\begin{abstract}
How can an individual have a sensory experience, very different of another person, receiving a similar sensory input? Developing an understanding of such differences and the mechanisms that support them is the base to optimize pain treatment on an individual-byindividual focus [1]. However, the scenario of chronic pain management is unsatisfactory, with many patients suffering from pain up to their maximum tolerance level, high rates of patient dissatisfaction, huge impact on their own quality of life and a large variability of pharmacological combinations used to treat it [2].
\end{abstract}

Chronic pain is an unpleasant sensory and emotional experience, without apparent biological value, that persists beyond normal tissue healing [3]. New opioid analgesic drugs appear to benefit from classical ones, in terms of efficacy and tolerability, but studies in 'real world' remain scarce, without any predictable biomarker of analgesic response [4]. The concept that 'one size fits all' has been replaced by the idea of patient-tailored healthcare prevention and therapy. This is the base of personalized medicine, which foresees the use of molecular data to better classify disease, to facilitate the development and validation of new targeted therapies, to treat patients with more specificity and efficacy but fewer adverse events and towards more accurately disease predisposition. So hopefully, patients with an unsatisfactory response to analgesic treatment will have a ray of hope [5].
Discovery efforts in pharmacogenomics (PGx) have been assisted by robust, nationally accessible reference databases which classify and annotate genomic variation, for example, single nucleotide polymorphism database/genomic structural variation database, ClinVar and Online Mendelian Inheritance in Man (OMIM) [6]. Genes can affect pharmacodynamics based on variations in drug target receptors and downstream signal transduction (i.e. OPRM1, COMT). On the other hand, genes affecting pharmacokinetics that affect drug metabolism and/or elimination (i.e. CYP450 family of enzymes, enzymes responsible for glucuronidation, drug transporter proteins and COX enzymes) altering the relationship between drug dose and steady-state serum drug concentrations $[7,8]$.

In musculoskeletal chronic pain, the contribution from the catecholaminergic system, represented by COMT and adrenoceptor $\beta 2$ (ADRB2), is seconded by the involvement of the serotonergic system, exemplified by associations between serotonin transporter (SLC6A4) and serotonin receptor 2A (HTR2A) [9]. Complementary nociceptive and inhibitory systems are implicated in postoperative pain studies: as the association between variant in GTP hydrolase, GCH1 (a regulator in the dopamine, serotonin and nitric oxide biosynthesis pathway) and postoperative pain [10] where OPRM1 genotypes could predict the extent of chronic postoperative pain, similarly as variants in

\section{Ana M Peiró}

Author for correspondence: Clinical Pharmacology Unit, Department of Health of Alicante, General Hospital, Alicante, Spain and

Neuropharmacology on Pain \& Functional Diversity (NED), Research Unit, Department of Health of Alicante, General Hospital, ISABIAL, Alicante, Spain

Tel.: +34965913868

peiro_ana@gva.es

\section{César Margarit}

Neuropharmacology on Pain \& Functional Diversity (NED), Research Unit, Department of Health of Alicante, General Hospital, ISABIAL, Alicante, Spain

and

Pain Unit, Department of Health of Alicante, General Hospital, Alicante, Spain

\section{Adrián LLerena}

CICAB Clinical Research Centre, Extremadura University Hospital \& Medical School, Badajoz, Spain 
$K C N S 1$, a voltage-gated potassium channel subunit [11]. Even more, genetic variants of inflammatory cytokines (IL1R2, and a haplotype within IL10), modulation of neurotransmission and related pathways as sodium channels (Nav1.7, and to a lesser extent Nav1.8 and Nav1.9) have been evidenced to play a role in postoperative neuropathic pain risk [12]. However, few knowledge resources are attempting to cross the 'omic chasm' of variant interpretation and delivery of evidenced-based clinical recommendations [13].

Next considerations are discussed: whether PGx test results are actionable; the level of evidence needed for implementation of PGx; the sources of information regarding the interpretation of PGx data and the need to move from local versions of national clinical guidelines to international ones. The rendering of clinical guidelines as structured, coded knowledge sources will facilitate their adoption and implementation by reducing the amount of expertise and effort required to extract and transform the knowledge from humanreadable forms into technical specifications for programming teams. Concerted efforts like an open active cooperation with industry are required in order to facilitate translation and commercialization exploring how to use genetic testing [14].

To move beyond, the successful adoption of PGx into routine clinical care requires different multidisciplinary consortiums and databases suitable for use in an electronic health record with clinical decision support [15]. In addition, payers need to be convinced about the positive cost benefit of PGx-guided healthcare, and healthcare professionals need to be trained

\section{References}

1 Cazacu I, Mogosan C, Loghin F. Safety issues of current analgesics: an update. Clujul. Med. 88(2), 128-136 (2015).

2 Pérez C, Margarit C, Serrano M; Spanish group of CHANGE PAIN patient survey. Survey of European patients assessing their own noncancer chronic pain: results from Spain. Curr. Med. Res. Opin. 29(6), 643-651 (2013).

3 Hardt J, Jacobsen C, Goldberg J, Nickel R, Buchwald D. Prevalence of chronic pain in a representative sample in the United States. Pain Med. 9, 803-812 (2008).

4 Langley P. The prevalence, correlates and treatment of pain in the European Union. Curr. Med. Res. Opin. 27, 463-480 (2011).

5 Webster LR, Belfer I. Pharmacogenetics and personalized medicine in pain management. Clin. Lab. Med. 36(3), 493-506 (2016).

6 Hoffman J, Dunnenberger H, Kevin Hicks J et al. Developing knowledge resources to support precision medicine: principles from the Clinical Pharmacogenetics Implementation Consortium (CPIC). J. Am. Med. Inform. Assoc. 23(4), 796-801 (2016). and reassured on the availability and utility of genomic testing. Maybe patients are one of the most important requirements for the new knowledge network envisaged [16]. Patients are better placed to demand the sharing of both data and professional credit that will be necessary to fully capture the value of this collaborative approach acquiring, synthesizing and widely disseminating biomedical knowledge, even more when 15\% of the medicinal products centrally approved in the $\mathrm{EU}$, since 1995, contain a genetic biomarker in their product label [17]. One of the more intriguing recent developments in cellular genomics is that naturally occurring noncoding miRNAs can profoundly inhibit gene expression by degrading mRNAs and regulating epigenetic machinery [18,19].

What is the future of PGx in pain management? A united group of professionals from governments, academia, private industry and caregivers turning PGx information in standard of care for anesthesiologists and pain management physicians [20]. Alan Kay, an UCLA computer scientist, affirms that "the best way to predict the future is to invent it."

\section{Financial \& competing interests disclosure}

The authors have no relevant affiliations or financial involvement with any organization or entity with a financial interest in or financial conflict with the subject matter or materials discussed in the manuscript. This includes employment, consultancies, honoraria, stock ownership or options, expert testimony, grants or patents received or pending, or royalties.

No writing assistance was utilized in the production of this manuscript.

7 Kirchheiner J, Schmidt H, Tzvetkov M et al. Pharmacokinetics of codeine and its metabolite morphine in ultra-rapid metabolizers due to CYP2D6 duplication. Pharmacogenomics J. 7, 257-265 (2007).

8 Kadiev E, Patel V, Rad P et al. Role of pharmacogenetics in variable response to drugs: focus on opioids. Expert Opin. Drug Metab. Toxicol. 4, 77-91 (2008).

9 Allegri M, De Gregori M, Minella CE et al.; PainOMICS Group. 'Omics' biomarkers associated with chronic low back pain: protocol of a retrospective longitudinal study. $B M J$ Open 16(10), e012070 (2016).

10 Kim DH, Dai F, Belfer I et al. Polymorphic variation of the guanosine triphosphate cyclohydrolase 1 gene predicts outcome in patients undergoing surgical treatment for lumbar degenerative disc disease. Spine (Phila Pa 1976) 35(21), 1909-1914 (2010).

11 Costigan M, Belfer I, Griffin RS et al. Multiple chronic pain states are associated with a common amino acidchanging allele in KCNS1. Brain 133(9), 2519-2527 (2010).

12 Waxman SG, Merkies IS, Gerrits MM et al. Sodium channel genes in pain-related disorders: phenotype-genotype 
associations and recommendations for clinical use. Lancet Neurol. 13(11), 1152-1160 (2014).

13 Starren J, Williams M, Bottinger E. Crossing the omic chasm: a time for omic ancillary systems. JAMA 309(12), 1237-1238 (2013).

14 Bank PC, Swen JJ, Guchelaar HJ. Pharmacogenetic biomarkers for predicting drug response. Expert Rev. Mol. Diagn. 14(6), 723-735 (2014).

15 Peiró AM, Planelles B, Juhasz G et al. Pharmacogenomics in pain treatment. Drug Metab. Pers. Ther. 31(3), 131-142 (2016).
16 Friedman C, Wong A, Blumenthal K. Achieving a nationwide learning health system. Sci. Transl. Med. 2, 57 (2010).

17 Ehmann F, Caneva L, Prasad K et al. Pharmacogenomic information in drug labels: European Medicines Agency perspective. Pharmacogenomics J. 15(3), 201-210 (2015).

18 Mogil J. Pain genetics: past, present and future. Trends Genet. 28(6), 258-266 (2012).

19 Niederberger E, Kynast K, Lötsch J et al. MicroRNAs as new players in the pain game. Pain 152, 1455-1458 (2011).

20 Desmond-Hellmann S. Toward precision medicine: a new social contract? Sci. Transl. Med. 4(129), 129ed3 (2012). 